\title{
Transmit-Receive Subarrays for MIMO Radar Array Antenna
}

\author{
Syahfrizal Tahcfulloh ${ }^{\mathrm{a},}{ }^{*}$ \\ ${ }^{a}$ Department of Electrical Engineering, Universitas Borneo Tarakan, Tarakan, 77123, Indonesia \\ Corresponding author: *rizalubt@gmail.com
}

\begin{abstract}
The phased multiple-input multiple-output (PMIMO) radar uses subarrays in transmit array that overlap one another to exploit the main advantage of the phased array (PA) radar, i.e., high directional coherent gain, and the main advantage of the MIMO radar, i.e., high waveform diversity gain. This paper has derived the radar formula that utilizes overlapping subarrays on transmit (Tx) and receive (Rx) array simultaneously called the transmit-receive subarrays of MIMO (TRSM) radar. The use of overlapping subarrays in Tx-Rx establishes the TRSM radar has high flexibility to configure the number of Tx-Rx subarrays that use the performance of all the gains simultaneously, so that produce Tx-Rx beampattern and signal-to-noise-plus-interference ratio (SINR) more than those achieved by PMIMO radars. The approach aims to overcome the beam shape loss, increase the transmit-receive gain, minimize the maximum peak sidelobe levels, narrow the half-power beamwidth, increase directivity, and increase SINR. The effectiveness of this radar's performance is compared to the PMIMO radar in various methods such as equal subarrays, unequal subarrays, and optimum partitioning, the PA, and the MIMO radar. The numerical simulation and evaluation results show that the proposed radar has several advantages such as lowest the peak sidelobe level, narrow the half-power beamwidth, and high directivity, so it is very resilient to interferences on target locations. The effect of the number of subarrays as a function of performance parameters on the Tx-Rx array that is ready to adjust to the detected target conditions is also presented.
\end{abstract}

Keywords - Array antenna; coherent gain; MIMO radar; phased array; transmit-receive subarray.

Manuscript received 26 Dec. 2019; revised 4 Jul. 2020; accepted 7 Aug. 2020. Date of publication 28 Feb. 2021. IJASEIT is licensed under a Creative Commons Attribution-Share Alike 4.0 International License.

\section{INTRODUCTION}

The phased array (PA) radar, with its beam steering capability, produces high directional coherent gain towards the target [1]. This coherent, directional gain is useful for detecting weak targets and minimizing sidelobe levels in the direction of interference [2]. Unlike the PA radar, the multiinput multi-output (MIMO) radar has omnidirectional transmission by all transmit (Tx) elements, which are orthogonal to each other so that they produce high waveform diversity gain. The MIMO radar equipped with signal processing can detect multiple targets [3]-[12], [23], [24]. However, the MIMO radar that is good in waveform diversity gain turns out poorly in the formation of coherent, directional gain. This problem can be overcome by using the PhasedMIMO (PMIMO) radar approach, which exploits the main advantage of the PA radar, i.e., coherent, directional gain, and the main advantage of the MIMO radar, i.e., waveform diversity gains simultaneously [13]. In addition, the PMIMO radar is still being studied to date because it has other advantages such as increasing the angular resolution, adding the identifiability parameters, expanding the aperture array, and expanding the target detection [13], [21].

In principle, the PMIMO radar is the MIMO radar whose elements of the Tx array are overlapped subarrays [13]-[16]. These subarrays are orthogonal to form directional beams such as the PA with the number of elements of the subarray in the Tx array being equal [13]-[16] and unequal [17]-[18]. The purpose of using Tx subarrays is to obtain high directional gain so that decreasing the maximum peak sidelobe level (MPSLL) is better than the PA radar. Although on the other hand, it causes beam shape loss on the transmitreceive beampattern ( $T-R$ beampattern). MPSLL is the value of the sidelobe level in $\mathrm{dB}$ for the first sidelobe of the main lobe or main beam. This also shows that when MPSLL on the PMIMO radar is low, directivity becomes large. The optimum partitioning method has obtained the optimum number of subarrays that have the lowest MPSLL and highest directional gain compared to the previous method [19]. However, the directivity gain obtained is not an optimum directivity. The optimum directivity produces half-power beamwidth (HPBW), which focuses on the intended target and, at the same time, increases the ability to suppress interference so 
that these conditions require the radar performance that has a high Tx-Rx gain as well as a high signal-to-interference-plusnoise ratio (SINR).

This paper presents a transmit-receive subarray approach to the MIMO radar (TRSM), where both the Tx and Rx arrays are divided into overlapping subarrays. Each subarray in the Tx-Rx array has the same number of elements. The variation of the waveform produced by combining the number of subarrays in the Tx-Rx array compensates for the beam shape loss and the directivity compared to when using only subarrays on the Tx array as in the PMIMO radar. An optimum variation of the waveform occurs simultaneously, increasing the coherent, directional gain and the waveform diversity gain. It has a significant effect on increasing the radar performance, such as low MPSLL, high directivity, narrow HPBW, and high SINR.

This paper extends the previous study about the effect of the number of subarrays in Tx array on MPSLL and beam shape loss [13]. In this case, the number of subarrays in the $\mathrm{Rx}$ array is also considered so that the number of subarrays in the Tx-Rx array provides optimum performance. The performance of the optimum number of subarrays is then compared with the radar performance investigated by [16] and [17]. The optimum number of subarrays from the PMIMO radar is $K / 2-1$ [16], $K$ is the total number of antenna elements in the Tx array, which provides the best radar performance. However, the Hybrid Phased MIMO radar with Unequal Subarrays (HPMR-US), it is obtained by subarray. The number of elements of each subarray varies [17].

Furthermore, it was also proved that with the optimum partitioning PMIMO radar (OPPM) [19], which produced a low MPSLL it did not provide optimum directivity. Optimum directivity determines the radar's HPBW and SINR. Overall, this paper shows the comparison of the performance of the MPSLL, the T-R beampattern, directivity, HPBW, and SINR Output between the TRSM radar and other radars. To clarify more, the advantages of the TRSM radar compared to other radars, the impact of the number of subarrays in Tx-Rx array on the performance of Tx-Rx gain, and SINR is also presented. This establishes the radar ready and able to adjust to any condition of the target to be detected.

\section{MATERIALS AND METHODS}

\section{A. Review of Subarray Methods in the Phased-MIMO Radar}

The PMIMO radar was introduced [13], which was originally designed to exploit the main advantage of the PA radar (i.e., directional gain) and the main advantage of the MIMO radar (i.e., waveform diversity gain). The approach is carried out by dividing the Tx array into several subarrays, which overlap with the number of equal elements of the antenna have been investigated by previous studies [13]-[16], and the number of elements of the antenna is unequal [17][18]. The PMIMO's illustration with equal subarray is shown in Fig. 1.

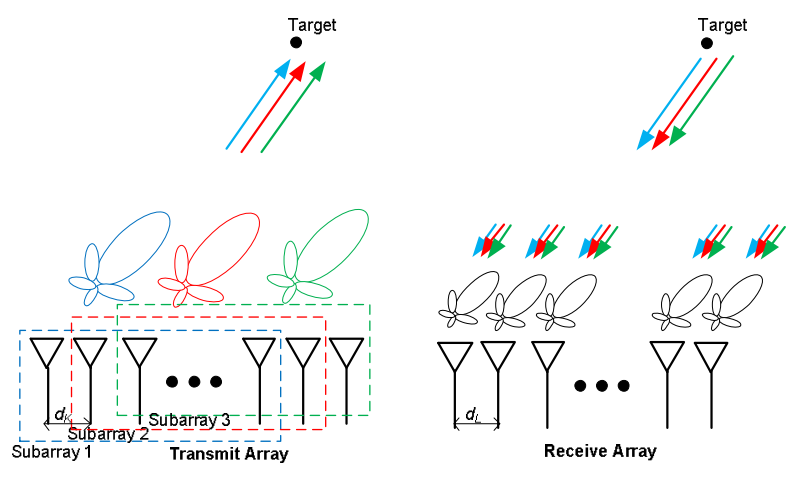

Fig. 1 Ilustration of the transmit-receive array for the PMIMO radar

The optimum number of subarrays $(M)$ [16] is $K / 2-1$ whereas the optimum number of subarrays of PMIMO radar is $(K / 2)-[(K-1) / 12$ [19]]. The radar performance in the term of T-R gain and SINR has increased when unequal subarray is used in the Tx array [17]. In summary, from the application of the three approaches, it is indicated that the selection of the right number of subarrays in the Tx array affects the MPSLL value, which at the same time impacts the main beam directivity and the HPBW.

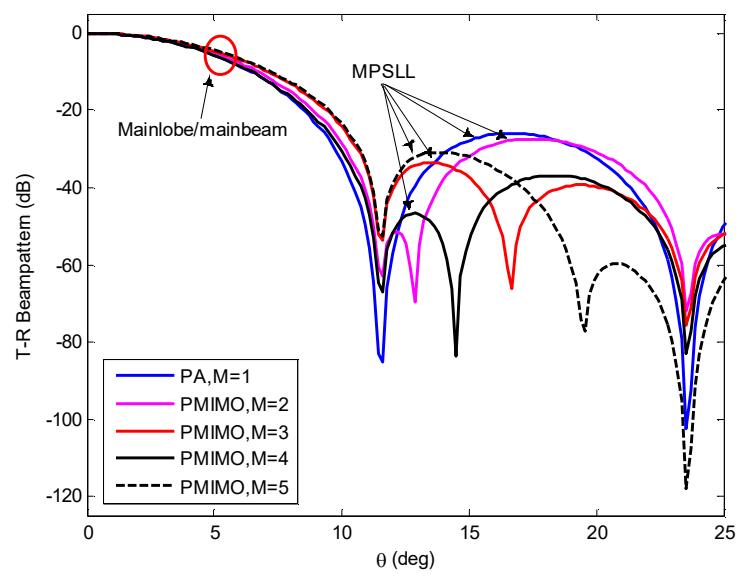

(a)

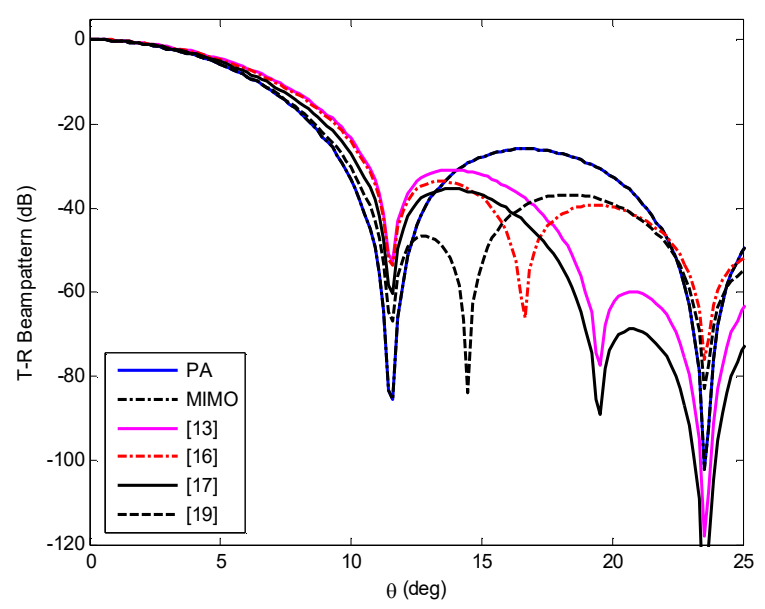

(b)

Fig. 2 T-R beampattern of the PMIMO radar with $K=L=10$ for: (a) variation of $M$ and (b) variety methods 
Fig. 2(a) is a simulation of T-R beam pattern from the PA and the PMIMO radar with varying number of subarrays starting from $M=1$ to $M=5$, the total number of antennas on Tx and Rx arrays is $K=L=10$ and the target at $\theta_{t}=0^{\circ}$. If it is desired that the MPSLL obtained is the lowest, and the directivity obtained from the main beam is narrow, or no beam shape loss occurs, then there must be a mechanism to determine the optimum number of subarrays. The lowest MPSLL value obtained by the PMIMO radar is $M=2$, but it has a smaller directivity than the PA radar. For the PMIMO radar with $M=5$ or the number of subarrays half of $K$, the MPSLL value is higher than the MPSLL value for PMIMO $(M=2)$. It can also be seen that the variation of the number of subarrays in Tx array proves that the lowest MPSLL is not only obtained by the number of subarrays, namely $K / 2-1$ [16], $(K / 2)-[(K-1) / 12]$ [19], and others. Therefore, it needed an effort that can compromise the number of subarrays to obtain radar performance with the lowest MPSLL without sacrificing its directivity, which is better than the PA radar. If the lowest MPSLL, high directivity, and narrow HPBW are achieved, then high SINR is also obtained.

The effect of the number of subarrays on the radar performance of various types of radar such as the PA, the MIMO, the PMIMO radar [13], the PMIMO radar as studied by [16] with a number of subarrays $M_{o}=K / 2-1$, the PMIMO radar as conceived by [17] with unequal subarrays, and the OPPM radar [19] with $M_{o}=(K / 2)-[(K-1) / 12]$ is shown in Fig. 2(b). In the OPPM method, it is obtained that MPSLL is indeed the lowest, but its directivity is still lower than the PA radar. This is because the PMIMO radar type utilizes a simultaneous compromise between directional coherent gain and waveform diversity gain only on the Tx array, while the $\mathrm{Rx}$ array does not have both gains. However, if the subarray method is also applied to Rx array, it has the potential to compensate for the lack of directivity or beam shape loss, so that produce high directivity while having a lower MPSLL. Numerical evaluations by [20], applying the use of subarray on Tx-Rx array, have shown that the Tx-Rx gain and SINR obtained significantly increase compared to the PMIMO radar performance. Thus, the various methods of the optimum subarray on the PMIMO radar with subarray only on Tx array in principle only produce main beam whose directivity is lower than the PA radar.

Fig. 3(a) shows the effect of the number of subarrays on the MPSLL of the PMIMO radar [13] with several of $K$. The number of subarrays determines the desired MPSLL. It also indicates that MPSLL can be adjusted to satisfy a performance criterion through an exact determination of the number of subarrays. The trend of the MPSLL curve obtained is like the MPSLL curve [19]. For Tx array with $K$ elements, there is some optimum number of subarrays $M$ with the lowest MPSLL following a certain formula [16], [19]. For example, for $K=\{49,50,51\}$ each has the lowest MPSSL of $-34.41 \mathrm{~dB}$ for two subarrays i.e. for $K=49$ at $M=12$ and $M=34$, for $K$ $=50$ at $M=14$ and $M=36$, and for $K=51$ at $M=16$ and $M$ $=38$. For the number of subarrays at $M=K / 2$, that is $M=\{24$, 25,26 ) of the various $K$ has a lower MPSLL of $4.5 \mathrm{~dB}$ than MPSLL the optimum number of the subarrays [19].

For the directivity value of the PMIMO radar with various $K$ shown in Fig. 3(b), it appears that when $M=K / 2$ has the lowest directivity value i.e. $41.6 \mathrm{~dB}$. Whereas at $K=49$ with
$M=12$ and $M=34, K=50$ with $M=14$ and $M=36$, and $K=$ 51 with $M=16$ and $M=38$ which have the lowest MPSLL it turns out that the directivity is $1 \mathrm{~dB}$ lower than directivity of the PA radar $(M=1$ for $K=50)$ i.e. $42.8 \mathrm{~dB}$.

For HPBW values of the three variations of $K$, namely 49 , 50, and 51, which are shown in Fig. 3(c), it appears that for $K$ $=50$ with $M=14$ and $M=36$ has a HPBW value i.e. $1.65^{\circ}$, while for $M=K / 2=25$ has a HPBW value i.e. $1.68^{\circ}$. As a comparison for the PA radar $(M=1$ for $K=50)$ it turns out to have an HPBW value, i.e., $1.47^{\circ}$. In summary, for optimum conditions of subarray with the lowest MPSLL has a relatively narrow HPBW and relatively large directivity. These conditions improve radar performance to improve the ability of target detection and suppress interference.

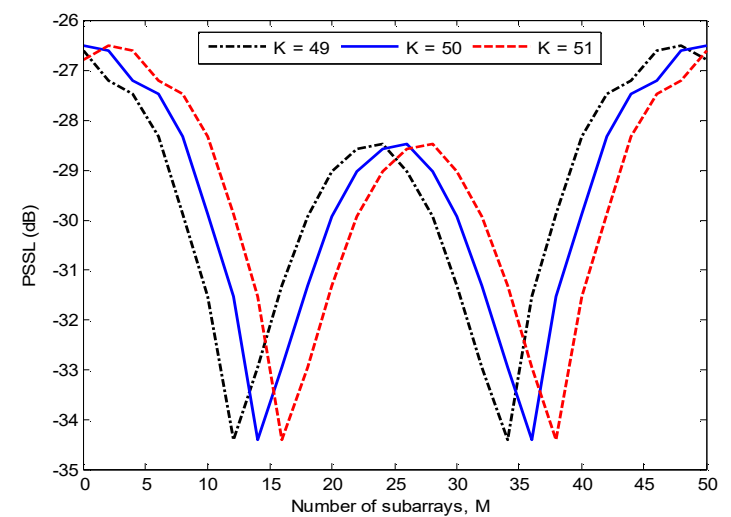

(a)

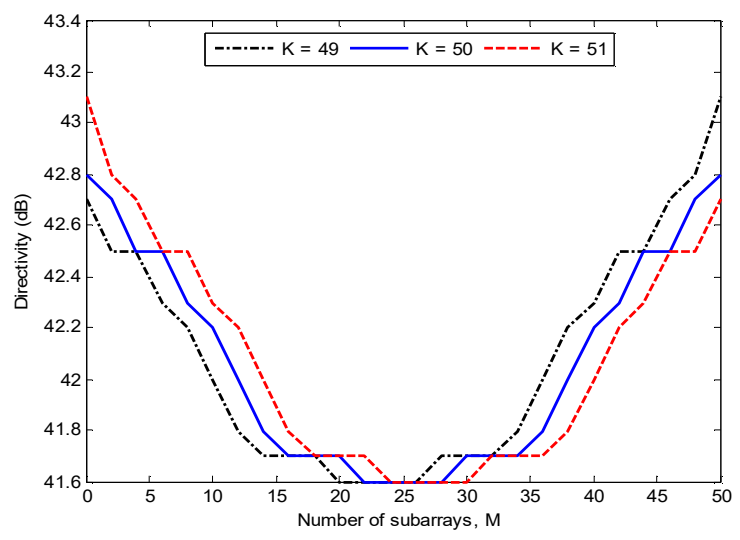

(b)

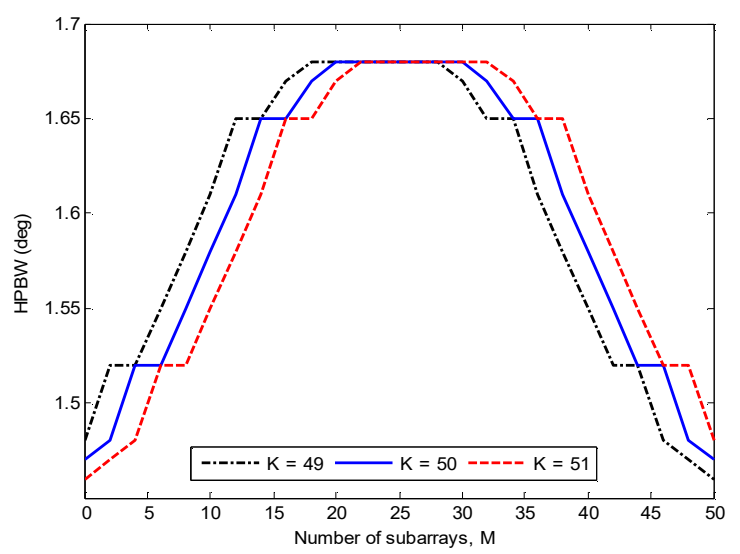

(c)

Fig. 3 The performance of the PMIMO radar against of $M$ at the variation of $K$ for (a) MPSLL, (b) directivity, and (c) HPBW 
HPBW in degree unit has a value that is inversely proportional to directivity. This means that high directivity radar has a narrow HPBW. It should be noted that a narrow HPBW, so-called pencil beam, is very useful if the desired radar works as the tracking radar. Based on the MPSLL, directivity, and HPBW values of the radar from an optimum number of subarrays, it is used as a basis for determining the optimum number of subarrays in the Tx-Rx subarrays method of the MIMO (TRSM) radar.

In the PMIMO radar [13], it was assumed that the radar system with collocated antennas had $K$ antenna on the Tx array and $L$ antenna on the Rx array. Spaces between Tx and $\mathrm{Rx}$ antenna elements are $d_{K}$ and $d_{L}$, respectively. The transmitted signal is assumed to be a narrowband signal, and its propagation is non-dispersive. The $\mathrm{Tx}$ array has been partitioned into $M$ subarray overlapping one another, as in Fig. 1 . The number of antenna elements in each subarray is $K-M$ +1 for the Tx array.

A beam is formed in each subarray in the direction of a certain target. Simultaneously each subarray on the Tx array emits a unique orthogonal waveform. The $m$-th subarray on the Tx array emits signals $\varphi_{m}(t)$ which are orthogonal to each other with other subarray signals. A beamforming weight vector is designed to maximize the coherent processing gain and waveform diversity gain on the subarray in the Tx array expressed by $M \times 1$ coherent vector transmit $\mathbf{c}(\theta)$ and vector transmit diversity $\mathbf{d}(\theta)$ as following [13]

$$
\begin{gathered}
\mathbf{c}(\theta)=\left[\begin{array}{llll}
\mathbf{w}_{1}^{H} \mathbf{a}_{1}(\theta) & \mathbf{w}_{2}^{H} \mathbf{a}_{2}(\theta) & \cdots & \mathbf{w}_{M}^{H} \mathbf{a}_{M}(\theta)
\end{array}\right]^{T} \\
\mathbf{d}(\theta)=\left[\begin{array}{llll}
e^{-j 2 \pi f \tau_{1}(\theta)} & e^{-j 2 \pi f \tau_{2}(\theta)} & \cdots & e^{-j 2 \pi f \tau_{M}(\theta)}
\end{array}\right]^{T}
\end{gathered}
$$

where $(\cdot)^{T}$ is a transpose operator, $(\cdot)^{H}$ is a Hermitian transpose operation, $\mathbf{w}_{m}$ is a unit-norm complex weight vector with $K$ elements for the $m$-th subarray on the Tx array which has $K-$ $M+1$ beamforming weights according to the active antenna element on the $m$-th subarray, $\mathbf{a}_{m}(\theta)$ is a $K \times 1$ transmit steering vector in the $m$-subarray, $f$ is the frequency of carrier signal, $\tau_{m}(\theta)$ is the relative delay of the first antenna element of the $m$-th subarray to the first element of the first subarray, $\tau_{m}(\theta)=m d_{K} \sin (\theta) / c$, and $c$ is the speed of light.

Furthermore, from the coherent transmit vector $\mathbf{c}(\theta)$ and diversity transmit vector $\mathbf{d}(\theta)$ obtained in the Rx array, the $M L$ $\times 1$ of Tx-Rx steering vector is expressed by [13]

$$
\mathbf{u}_{\text {PMIMO }}(\theta)=(\mathbf{c}(\theta) \circ \mathbf{d}(\theta)) \otimes \mathbf{b}(\theta)
$$

where $\circ$ represents the Hadamard product, $\otimes$ is the Kronecker multiplication operator, and the receive steering vector $\mathbf{b}_{n}(\theta)$ is similar to $\mathbf{a}_{m}(\theta)$. All vectors in $\mathbf{u}_{\text {PMIMO }}(\theta)$ namely $\mathbf{c}(\theta), \mathbf{d}(\theta)$, and $\mathbf{b}(\theta)$ are very dependent on the number of subarrays $M$ on the Tx array. For radar [13], the number of subarrays is $K / 2$, whereas $(K / 2)-1[16]$. In HPMR-US [17], the number of subarrays is unequal, and between subarray does not have a phase difference so that the diversity vector transmit component $\mathbf{d}(\theta)$ in (3) is $\mathbf{1}$, and the OPPM radar [19] has the number of subarrays expressed by $(K / 2)-[(K-1) / 12]$. It also explains that the number of subarrays determines all radar performance on various PMIMO radars only in the Tx array, which is $M$.

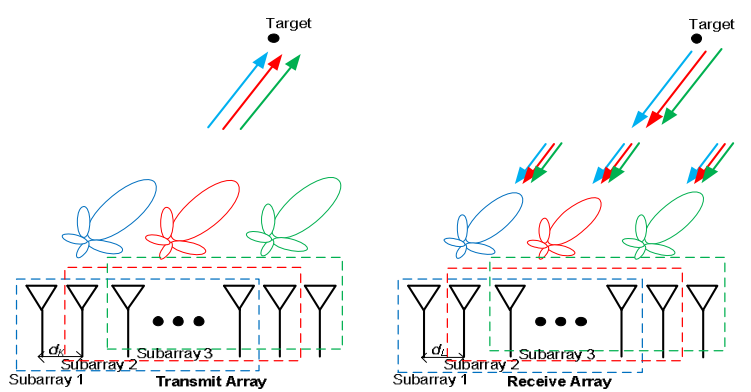

Fig. 4 Illustration of transmit-receive array for TRSM radar with $M=N=3$

B. Antenna Arrays of the MIMO Radar with TransmitReceive Subarrays

The weakness of various PMIMO radar methods with the subarray technique only in the Tx array is the existence of beam shape loss so that the directivity and HPBW values are lower than on the PA radar. Whereas the directivity and HPBW greatly influence the determination of MPSLL and the ability to minimize interference.

To overcome this weakness, the Tx-Rx subarrays approach for MIMO radar (TRSM) is proposed, which utilizes a combination of overlapping subarray simultaneously on Tx and $\mathrm{Rx}$ array. Each subarray in the Tx-Rx array is equal. The waveform variation produced by the combination of the number of subarrays in the Tx-Rx array compensates for the beam shape loss and directivity. The optimum combination of waveform simultaneously increases coherent, directional gain and waveform diversity gain, so it impacts improvements in the radar performance such as minimum MPSSL, high directivity, narrow HPBW, and high SINR.

It is assumed that a radar system with collocated antennas has $K$ antenna on the Tx array and $L$ antenna on the Rx array as in Figure 4. Spaces between Tx and Rx antenna elements are $d_{K}$ and $d_{L}$, respectively. Tx and $\mathrm{Rx}$ arrays have been partitioned into $M$ and $N$ subarrays, which overlap one another. The number of antenna elements in each subarray is $K-M+$ 1 for the Tx array and $L-N+1$ for the $\mathrm{Rx}$ array. Each subarray works as a PA, so that each one forms a beam that goes in a certain direction. A beamforming weight vector is designed so as to maximize the coherent processing gain and waveform diversity gain on the subarray on the Tx array and also the Rx array expressed by $M \times 1$ coherent transmit vector $\mathbf{c}(\theta)$ and the transmit diversity vector $\mathbf{d}(\theta)$ and also $N \times 1$ receive coherent vector $\mathbf{e}(\theta)$ and diversity transmit vector $\mathbf{f}(\theta)$ to produce the $M N \times 1$ of T-R steering vector expressed by [22]

$$
\mathbf{u}_{\mathrm{TRSM}}(\theta)=(\mathbf{c}(\theta) \circ \mathbf{d}(\theta)) \otimes(\mathbf{e}(\theta) \circ \mathbf{f}(\theta))
$$

with

$$
\begin{gathered}
\mathbf{e}(\theta)=\left[\begin{array}{llll}
\mathbf{v}_{1}^{H} \mathbf{b}_{1}(\theta) & \mathbf{v}_{2}^{H} \mathbf{b}_{2}(\theta) & \cdots & \mathbf{v}_{N}^{H} \mathbf{b}_{N}(\theta)
\end{array}\right]^{T} \\
\mathbf{f}(\theta)=\left[\begin{array}{llll}
e^{-j 2 \pi f \tau_{1}(\theta)} & e^{-j 2 \pi f \tau_{2}(\theta)} & \cdots & e^{-j 2 \pi f \tau_{N}(\theta)}
\end{array}\right]^{T}(6)
\end{gathered}
$$

where $\mathbf{v}_{n}$ is the $L \times 1$ unit-norm complex weight vector for the $n$-th subarray in the Rx array with a definition similar to $\mathbf{w}_{m}$, $\mathbf{e}(\theta)$ is the $L \times 1$ receive coherent vector, and $\mathbf{f}(\theta)$ is the $L \times 1$ receive vector diversity. Unlike (3), in (4) indicates that all the performance of the TRSM radar is determined not only by the number of subarrays in the Tx array, but also the number of subarrays in the Rx array i.e. $M$ and $N$, respectively. 
In general, the performance of the TRSM radar is expressed in Tx-Rx gain and SINR output. For the Tx-Rx gain of the TRSM radar is formulated with

$$
G(\theta)=(K / M)\left|\mathbf{w}_{r}^{H} \mathbf{u}_{\mathrm{TRSM}}(\theta)\right|^{2}
$$

If it is assumed that $\mathbf{w}_{r}=\mathbf{u}_{\text {TRSM }}(\theta)$ then (7) becomes

$$
G(\theta)=(K / M)\left|\mathbf{u}_{\mathrm{TRSM}}^{H}(\theta) \mathbf{u}_{\mathrm{TRSM}}(\theta)\right|^{2}
$$

For a uniform linear arrays (ULAs) then $\left|\mathbf{a}_{M}{ }^{H}(\theta) \mathbf{a}_{M}(\theta)\right|=K$ $-M+1,\left|\mathbf{d}_{M}{ }^{H}(\theta) \mathbf{d}_{M}(\theta)\right|=M,\left|\mathbf{b}_{N}{ }^{H}(\theta) \mathbf{b}_{N}(\theta)\right|=L-N+1$, and $\left|\mathbf{f}_{N}{ }^{H}(\theta) \mathbf{f}_{N}(\theta)\right|=N$ then (8) is simplified to

$$
G=(K-M+1)^{2}(L-N+1)^{2} K M N^{2}
$$

The SINR output of the TRSM radar expressed by

$$
\operatorname{SINR}=\frac{(K / M) \sigma_{l}^{2}\left|\mathbf{w}_{r}^{H} \mathbf{u}_{\mathrm{TRSM}}(\theta)\right|^{2}}{\mathbf{w}_{r}^{H} \mathbf{R}_{i+n} \mathbf{w}_{r}}
$$

where $\mathbf{R}_{i+n}$ is the matrix of covariance interference plus noise. For the case of dominant noise power, like getting (9) then (10) is simplified into

$$
S N R=(K-M+1)(L-N+1) K N\left(S N R_{o}\right)
$$

and if the case of dominant interference power, then (10) becomes

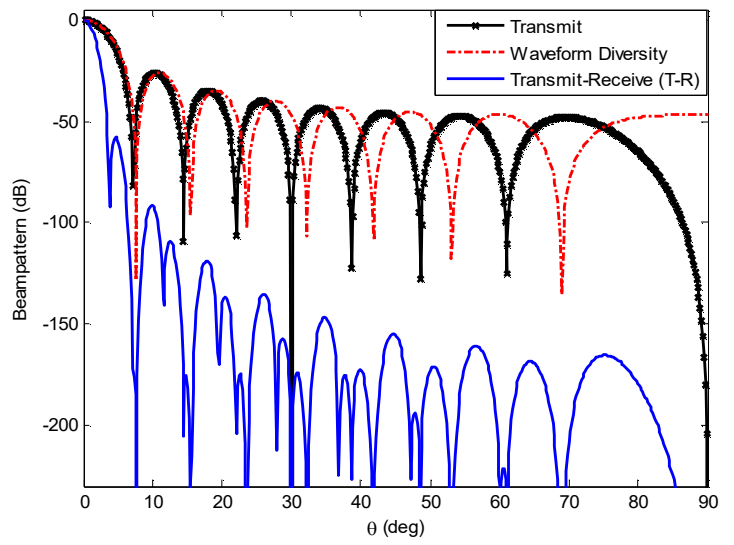

(a)

$$
S I R=(K-M+1)(L-N+1) M N\left(S I R_{o}\right)
$$

where SNRo and SIRo are the minimum value that the radar system has about $10 \mathrm{~dB}$ [22].

\section{RESULTS AND DISCUSSION}

In this simulation, it is assumed that the number of transmitter and receiver antennas is the same $(K=L)$ with the number of subarrays in Tx and Rx arrays $1<M<K$ and $1<$ $N<L$, respectively. The distance between the antenna elements in the transmitter-receiver is a half wavelength. A target angle $\theta_{t}=0^{\circ}$. Noise is Gaussian with zero mean spatially and has the same variance on each antenna element. The performance of the T-R gain and SINR output on TRSM radar use (8) and (10), respectively, to show their beam pattern. Meanwhile, to investigate the effect of the number of subarrays in the Tx-Rx array on the radar performance using (9) $-(12)$.

\section{A. Impact of the Total Number of Antennas to T-R Beampattern}

For beam pattern on the TRSM radar with $K=L=30$ and $M_{\mathrm{o}}=N_{\mathrm{o}}=15$, starting from transmitting, waveform diversity, and transmit-receive shown in Fig. 5(a). It appears that null is at a certain angle on the transmit beampattern related to the sidelobe on the waveform diversity beampattern.

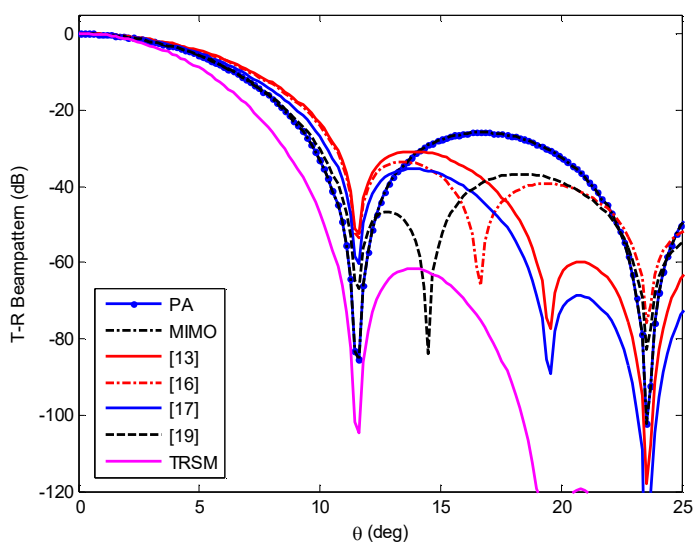

(b)

Fig. 5 Beampattern of the TRSM radar for (a) transmit, waveform diversity and T-R beampattern with $K=L=30$ and (b) T-R beampattern for all radars with $K$ $=L=10$

This also indicates that if the null is at a certain angle on the waveform diversity, beampattern means it is related to sidelobe on the transmit beam pattern. Both conditions yield null on the T-R beampattern. Thus, when setting null either on transmit or waveform diversity beam pattern, it gives null on the T-R beam pattern, which determines the ability to minimize interference on the target location. In Fig. 5(b), all the PMIMO radar models using only the subarray in the Tx array have the beamwidth of the main beam tending to be wider than the beamwidth of the PA radar. Although all these radar models have MPSLL is much lower than the PA radar. Unlike the case with the TRSM radar, which has a beam with much narrower than all the PMIMO radar models, especially the PA radar. Likewise, the TRSM radar has the lowest MPSLL compared to all types of radars. So, applying subarray on Rx array to compensate for the weakness of the beam shape loss from transmit coherent, directional gain and transmit diversity gain. In summary, the use of subarray methods on Tx and Rx array simultaneously produces the beamwidth of the main beam with the lowest MPSLL without sacrificing its directivity.

\section{B. SINR Output}

Fig. 6(a) shows the SINR output versus the INR for all types of radars with varying INR values $(I N R=S N R)$. When the SNR is low, the SINR output of the PA radar is higher than the SINR output of the MIMO radar. Furthermore, when the SNR value increases, the difference in SINR output from the PA and the MIMO radars tends to decrease, leading to the same value [13]. For the OPPM radar, it has a higher SINR 
output when SNR is low, i.e., $\mathrm{SNR}=\mathrm{INR}=10 \mathrm{~dB}$ compared to the SINR output of the TRSM radar. However, after SNR $=\mathrm{INR}=30 \mathrm{~dB}$, the SINR output of the TRSM radar is more significant than $10.58 \mathrm{~dB}$ compared to the SINR output of the OPPM radar. This shows that the SINR output performance

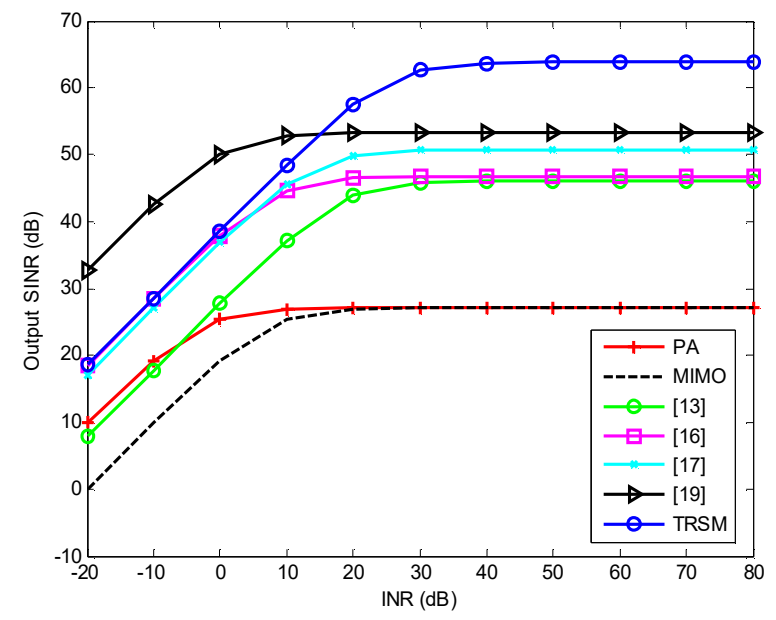

(a) of the TRSM radar becomes maximum suppressing interference when the interference power condition is greater than $30 \mathrm{~dB}$ and vice versa if the interference power condition is lower than $10 \mathrm{~dB}$, then the ability to minimize the interference is low.

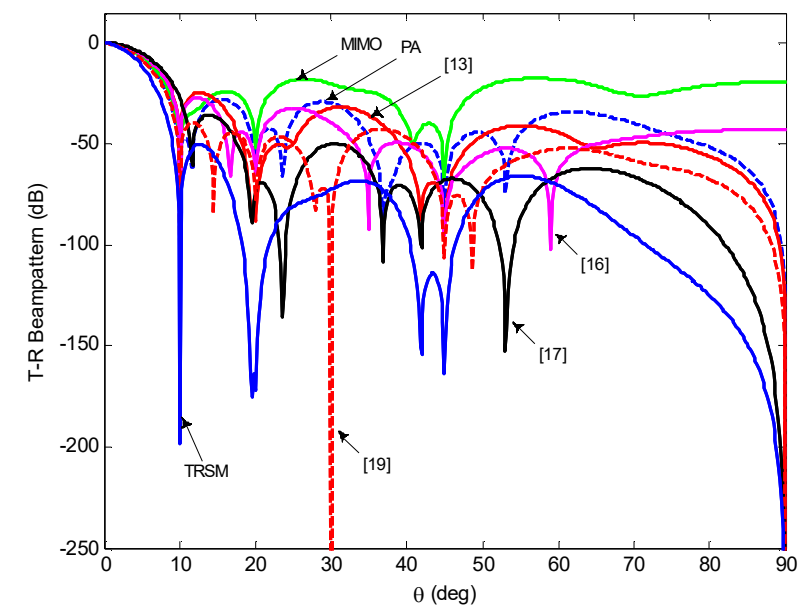

(b)

Fig. 6 Performance of all radars for (a) Output SINR versus INR = SNR and (b) T-R beampattern using MVDR beamformer

Also seen from Fig. 6(a) that for the optimum number of subarrays $M=K / 2-1[16]$ it turns out that the SINR output values are almost the same between the PMIMO radar [13] after SNR greater than $40 \mathrm{~dB}$.

\section{Beampattern with the Adaptive Beamformer}

To complete the discussion on the performance of PMIMO radar models, especially in dealing with interference, a T-R beampattern of all types of radar is presented using the MVDR (minimum variance distortionless response) beamformer [13], [17],[18]. In Fig. 6(b) shows the T-R beampattern of all types of radars with a target location of $0^{\circ}$ and some interference locations i.e., $10^{\circ}, 20^{\circ}$, and $45^{\circ}$. The target power is $0 \mathrm{~dB}$, and the interference power is $30 \mathrm{~dB}$. Appear at the locations of interference are all types of radars providing a nulling effect to suppress the smallest possible effect. The performance of the TRSM radar shows the superiority of all types of radars with the lowest MPSLL. This means that the performance of the TRSM radar is very robust against the presence of interference and at the same time, also utilizes the advantages of Tx-Rx waveform diversity gain.

\section{Performance of MPSLL, HPBW, and Directivity}

Table I shows a comparison between all types of radar in terms of MPSLL, directivity, and HPBW for various $K$. It appears that the overall performance of the TRSM radar compared to the PMIMO radar in [13], the PMIMO radar ( $M$ $=K / 2-1)$ in [16], the HPMR-US radar in [17], the OPPM radar in [19] has MPSLL, directivity, and HPBW much better than other types of radars. The average MPSLL value on the TRSM radar is twice the MPSLL value on the PMIMO radar because the TRSM radar utilizes directional coherent and waveform diversity gain simultaneously in Tx array (such as the PMIMO radar) and in Rx array.

TABLE I

COMPARISON BETWEEN TRSM AND ANOTHER RADAR IN MPSLL, DIRECTIVITY, AND HPBW

\begin{tabular}{|c|c|c|c|c|c|c|c|c|c|c|c|c|c|c|c|}
\hline \multirow[b]{2}{*}{$\boldsymbol{K}$} & \multicolumn{5}{|c|}{ MPSLL (dB) } & \multicolumn{5}{|c|}{ Directivity (dB) } & \multicolumn{5}{|c|}{ HPBW (deg) } \\
\hline & {$[13]$} & [16] & [17] & [19] & TRSM & {$[13]$} & {$[16]$} & [17] & [19] & TRSM & [13] & [16] & [17] & [19] & TRSM \\
\hline 10 & -30.8 & -33.8 & -35.4 & -46.7 & -61.7 & 27.8 & 27.9 & 28.8 & 29.0 & 30.9 & 8.30 & 8.16 & 7.40 & 7.20 & 5.80 \\
\hline 20 & -29.4 & -30.1 & -33.9 & -37.3 & -58.8 & 33.8 & 33.8 & 34.4 & 34.6 & 36.4 & 4.18 & 4.14 & 3.88 & 3.80 & 3.06 \\
\hline 30 & -28.9 & -28.2 & -33.4 & -35.1 & -57.9 & 37.3 & 37.4 & 37.9 & 38.1 & 40.2 & 2.78 & 2.74 & 2.60 & 2.54 & 1.98 \\
\hline 40 & -28.8 & -28.7 & -32.9 & -34.9 & -57.3 & 39.7 & 39.7 & 40.3 & 40.5 & 42.6 & 2.10 & 2.10 & 1.96 & 1.92 & 1.50 \\
\hline 50 & -28.6 & -28.6 & -32.7 & -34.7 & -57.0 & 41.6 & 41.8 & 42.2 & 42.4 & 44.6 & 1.68 & 1.66 & 1.58 & 1.54 & 1.20 \\
\hline 60 & -28.5 & -28.4 & -32.6 & -34.7 & -57.0 & 43.2 & 43.4 & 43.9 & 44.6 & 46.2 & 1.40 & 1.38 & 1.30 & 1.20 & 1.00 \\
\hline 70 & -28.4 & -28.3 & -32.7 & -34.8 & -56.9 & 44.6 & 44.6 & 45.2 & 45.3 & 47.5 & 1.20 & 1.20 & 1.12 & 1.10 & 0.86 \\
\hline 80 & -28.3 & -28.3 & -32.5 & -34.5 & -56.7 & 45.7 & 45.7 & 46.3 & 46.5 & 48.8 & 1.05 & 1.05 & 0.98 & 0.96 & 0.74 \\
\hline 90 & -28.2 & -28.2 & -32.4 & -34.4 & -56.4 & 46.6 & 46.6 & 47.2 & 47.3 & 49.6 & 0.95 & 0.95 & 0.89 & 0.88 & 0.67 \\
\hline 100 & -28.2 & -28.2 & -33.8 & -34.4 & -56.4 & 47.6 & 47.6 & 48.7 & 48.9 & 50.4 & 0.85 & 0.85 & 0.75 & 0.74 & 0.61 \\
\hline
\end{tabular}

In general, the number of elements, $K=L$, appears to increase, so the MPSLL value tends to decrease for all types of radars. This tendency gives meaning to the demands of high MPSLL levels, so the number of antenna elements in the Tx-Rx array should not be large. Another result from Table I, which is also important, is that the TRSM radar has a low MPSLL without sacrificing directivity from the main beam. Thus, with $K$ increasing, the value of MPSLL tends to decrease, the value of directivity tends to increase, and the value of HPBW tends to decrease. 


\section{E. Impact of the Number of Subarrays}

The performance evaluation of the T-R, SNR, and SIR gain for various configurations of the number of subarrays on the TRSM radar is presented in Figs. 7(a)-(c). Evaluation of these performances uses (9), (11), and (12) for $K=L=50$. Examples for special configurations of TRSM radar are the PA, the MIMO, and the PMIMO radar. The results of numerical performance evaluations for various configurations are presented in Table II. These results include radar performance such as T-R, SIR, and SNR gain for the PA, the MIMO, and the PMIMO radars [13]. According to [13] that the T-R gain of the PA radar is the same as the T-R gain of MIMO radar through numerical results, $G_{\mathrm{PA}}=G_{\mathrm{MIMO}}=67.96$ $\mathrm{dB}$, has been validated. Similarly, the $\mathrm{SNR}$ gain, $S N R_{\mathrm{PA}}=$ $M \cdot S N R_{\mathrm{MIMO}}$ [13]. For $M=50$ then $S N R_{\mathrm{PA}}=50.97 \mathrm{~dB}$ while $S N R_{\mathrm{MIMO}}=33.98 \mathrm{~dB}$ so that it is proven that $S N R_{\mathrm{PA}}=$ $M \cdot S N R_{\mathrm{MIMO}}$. Likewise, according to [13] that $S I R_{\mathrm{PA}}=S I R_{\mathrm{MIMO}}$ is also proven.

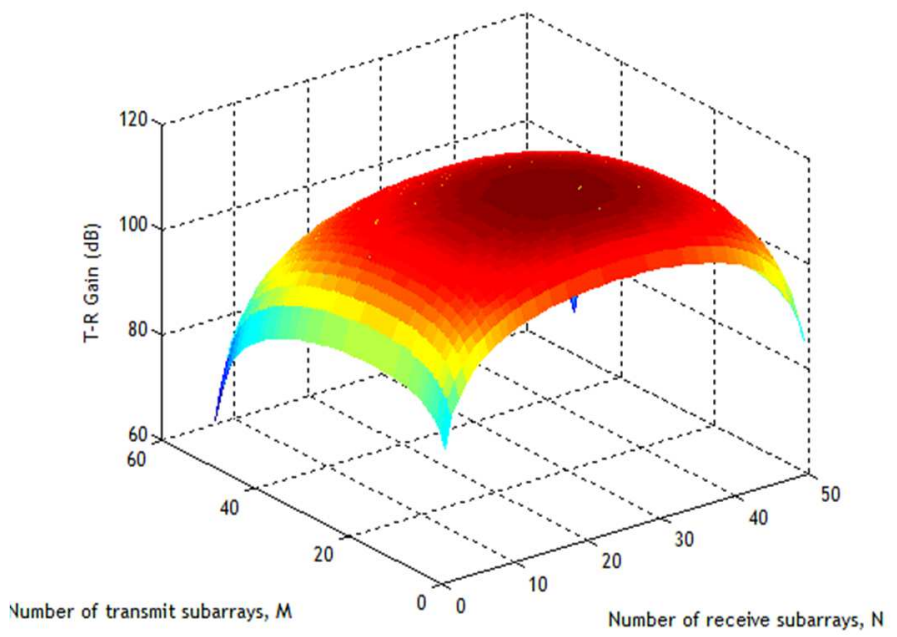

(a)

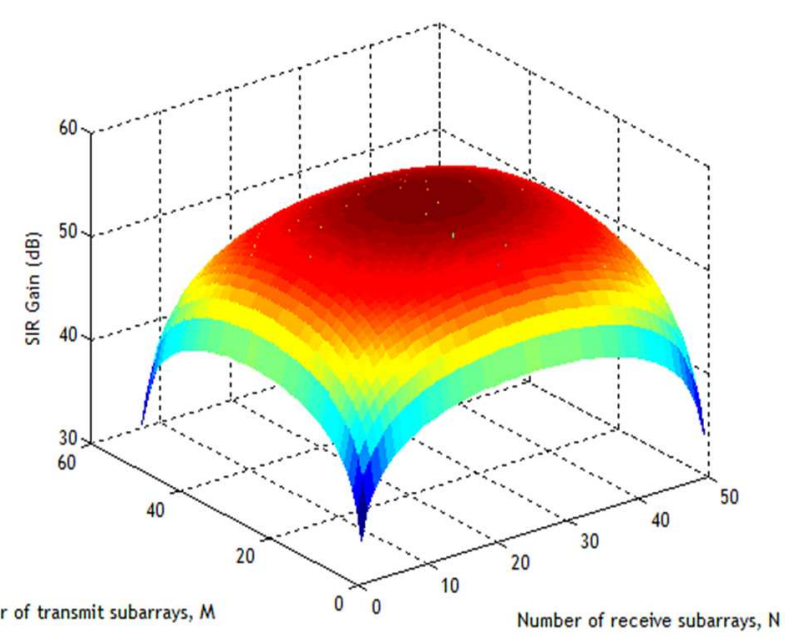

(b)

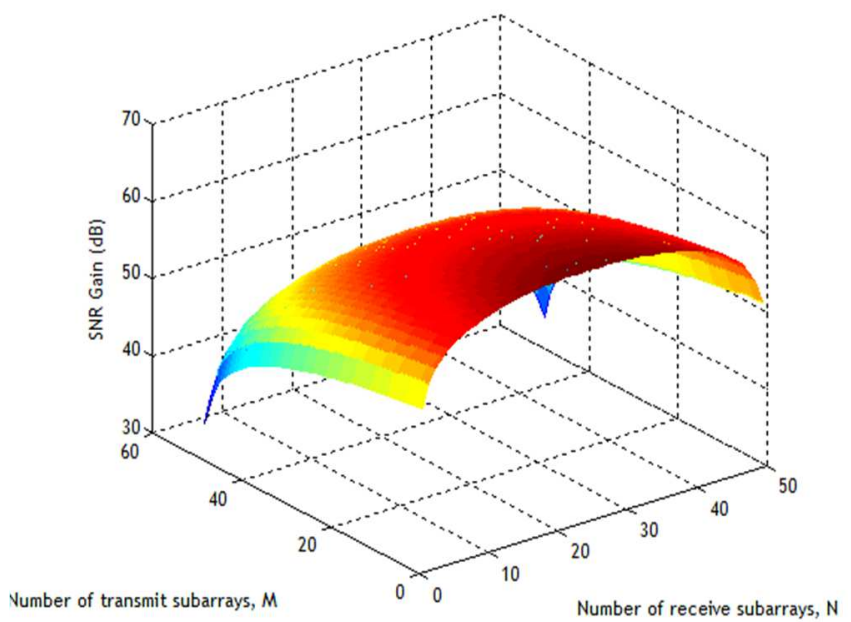

(c)

Fig. 7 The performance of the TRSM radar against of $M$ and $N$ at $K=L=50$ for: (a) T-R, (b) SIR, and (c) SNR gain

TABLE II

PERFORMANCE COMPARISON OF VARIOUS CONFIGURATION FOR THE TRSM RADAR IN $M$ AND $N$ WITH $K=L=50$

\begin{tabular}{ccc}
\hline Configuration & T-R gain (dB) & SNR gain (dB) \\
\hline$M=1, N=50$ (PA) & 67.96 & 50.97 \\
$M=N=50$ (MIMO) & 67.96 & 33.98 \\
$M=25, N=50$ (PMIMO in [13]) & 90.24 & 48.13 \\
$M=N=25$ & 112.52 & 59.27 \\
$M=1, N=25$ & 90.24 & 62.11 \\
$M=50, N=25$ & 90.24 & 45.12 \\
$M=1, N=1$ & 67.96 & 50.97 \\
$M=25, N=1$ & 90.24 & 48.13 \\
$M=50, N=1$ & 67.96 & 33.98 \\
$M=24, N=50$ (PMIMO in $[16])$ & 93.40 & 48.29 \\
$M=7, N=50$ (HPMR-US in $[17])$ & 92.29 & 45.12 \\
$M=20, N=50$ (OPPM in [19]) & 93.81 & 33.98 \\
\end{tabular}


Table II also shows that the TRSM radar has high flexibility to adapt to all performance based on various desired target conditions. This fact is also supported by the calculation of T-R gain for variants of PMIMO radar, such as the PMIMO radar [16], HPMR-US [17], and OPPM [19]. Another calculation result is the comparison of radar performances, T-R and SINR gain, between the TRSM radar $(M=N=25)$ against the PMIMO radar $(M=25, N=50)$, i.e., an increase in T-R and SINR gain by a factor $(N / L)^{2} L N^{2}$ and $(N / L) L N$, respectively.

In general, radar configurations with subarrays in either $\mathrm{Tx}$ or Rx array incline to produce higher gain than without using it. This is shown in Figs. 7(a) and 7(b) for the performance of the T-R and SIR gain. Despite the fact that the SNR gain performance with a subarray configuration $(M=N=25)$ is not a configuration that provides optimum performance (see Fig. 7(c)). For example, a radar configuration with $(M=1, N$ $=25$ ) has the highest SNR gain rather than to other configurations, which is $62.11 \mathrm{~dB}$. This also indicates that a radar configuration having a high SNR does not necessarily provide the other performance as well.

\section{CONCLUSION}

In this paper, a formula for Tx-Rx subarrays at the MIMO radar (TRSM) has been formulated, including performance parameters, especially Tx-Rx gain, SINR, MPSLL, directivity, and HPBW. Determination of the optimum Tx subarray $(M)$, which affects the MPSLL, directivity, and HPBW for various $K$ has also been presented and evaluated. The use of subarray methods on Tx and Rx arrays, i.e., $M$ and $N$ simultaneously on the TRSM radar, generates beamwidth from the main beam with the lowest MPSLL without compromising its directivity so that a high SINR output is produced compared to other types of radars. The high flexibility of the TRSM radar can make it easier to detect various conditions of the target and its environment. This can be realized through adjustments to the number of subarrays ( $M$ and $N$ ) in Tx-Rx array so that it will simplify the implementation and design of the radar system.

\section{REFERENCES}

[1] M. I. Skolnik, Introduction to Radar Systems, $3^{\text {rd }}$ ed. New York, NY, USA: McGraw-Hill, 2001.

[2] M. S. Davis and A. D. Lanterman, "Coherent MIMO Radar: The Phased Array and Orthogonal Waveforms," IEEE Aerosp. Electron. Syst. Mag., vol. 29, no. 8, pp. 76-91, Aug. 2014.

[3] A. Haimovich, R. Blum, and L. Cimini, "MIMO Radar with Widely Separated Antennas," IEEE Signal Process. Mag., vol. 25, no. 1, pp. 116-129, Jan. 2008.

[4] D. R. Fuhrmann and G. S. Antonio, "Transmit Beamforming for MIMO Radar Systems using Signal Cross-Correlation," IEEE Trans. Aerosp. Electron. Syst., vol. 44, no. 1, pp. 171-186, Jan. 2008.

[5] Y. Li, S. Gu, and N. Zheng, "MIMO Radar Transmit Beampattern Design for DOA Estimation with Sidelobe Suppression," Int. J. Antennas Propag., vol. 2016, Article ID 1512843, 10 pages, 2016.
[6] J. Li, P. Stoica, X. Luzhou, and W. Roberts, "On Parameter Identifiability of MIMO Radar," IEEE Signal Process. Lett., vol. 14, no. 12 , pp. $968-971$, Dec. 2007.

[7] G. Zheng and J. Tang, "Two-Dimensional DOA Estimation for Monostatic MIMO Radar with Electromagnetic Vector Received Sensors," Int. J. Antennas Propag., vol. 2016, Article ID 2952539, 10 pages, 2016.

[8] X. Song, N. Zheng, and T. Bai, "Resource Allocation Schemes for Multiple Targets Tracking in Distributed MIMO Radar Systems," Int. J. Antennas Propag., vol. 2017, Article ID 7241281, 12 pages, 2017.

[9] K. Gao, H. Shao, J. Cai, H. Chen, and W. Q. Wang, "Frequency Diverse Array MIMO Radar Adaptive Beamforming with RangeDependent Interference Suppression in Target Localization," Int. J. Antennas, Propag., vol. 2015, Article ID 358582, pp. 1-10, Sep. 2015.

[10] I. Pasya, N. Iwakiri, and T. Kobayashi, "Joint Direction-of-Departure and Direction-of-Arrival Estimation in a UWB MIMO Radar Detecting Targets with Fluctuating Radar Cross Sections," Int. J. Antennas Propag., vol. 2014, Article ID 847815, 15 pages, 2014.

[11] A. Hassanien and S. A. Vorobyov, "Transmit/Receive Beamforming for MIMO Radar with Colocated Antennas," in IEEE Int. Conf. Acoust. Speech Signal Process., Taipei, Taiwan, Apr. 2009, pp. 2089-2092.

[12] D. Wilcox and M. Sellathurai, "On MIMO Radar Subarrayed Transmit Beamforming," IEEE Trans. Signal Process., vol. 60, no. 4, pp. 2076-2081, Apr. 2012.

[13] A. Hassanien and S. A. Vorobyov, "Phased-MIMO Radar: A Tradeoff between Phased-Array and MIMO Radars," IEEE Trans. Signal Process., vol. 58, no. 6, pp. 3137-3151, Jun. 2010.

[14] W. Q. Wang, "Phased-MIMO Radar with Frequency Diversity for Range-Dependent Beamforming," IEEE Sensors J., vol. 13, no. 4: pp. 1320-1328, Apr. 2013.

[15] D. R. Fuhrmann, J. P. Browning, and M. Rangaswamy, "Signaling Strategies for the Hybrid MIMO Phased-Array Radar," IEEE J. Sel. Topics Signal Process., vol. 4, no. 1, pp. 66-78, Feb. 2010.

[16] N. E. D. Ismail, S. H. Mahmoud, A. S. Hafez, and T. Reda, "A New Phased MIMO Radar Partitioning Schemes," in IEEE Aerosp. Conf., Big Sky, Montana, Mar. 2014, pp. 1-7.

[17] W. Khan, I. M. Qureshi, A. Basit, and M. Zubair, "Hybrid Phased MIMO Radar with Unequal Subarrays," IEEE Antennas Wireless Propag. Lett., vol. 14, pp. 1702-1705, Apr. 2015.

[18] S. Tahcfulloh and G. Hendrantoro, "Phased-MIMO Radar using Hadamard Coded Signal," in IEEE Int. Conf. Radar Antenna Microw. Electron. Telecommun., Tangerang, Indonesia, Oct. 2016, pp. 13-16.

[19] A. Alieldin, Y. Huang, and W. M. Saad, "Optimum Partitioning of a Phased-MIMO Radar Array Antenna," IEEE Antennas Wireless Propag. Lett., vol. 16, pp. 2287-2290, 2017.

[20] S. Tahcfulloh and G. Hendrantoro, "Phased MIMO Radar with Coherent Receive Arrays," in IEEE Int. Conf. Signal Syst, Bali, Indonesia, May 2017, pp. 72-76.

[21] T. D. Backes, "Parameter Identifiability in Phased-Subarray MIMO Radar," in IEEE Aerosp. Conf., Big Sky, Montana, Mar. 2014, pp. 16.

[22] S. Tahcfulloh and G. Hendrantoro, "Full Phased MIMO Radar with Colocated Antennas," Int. J. on Commun. Antenna and Propag., vol. 9, no. 2, pp. 144-154, Apr. 2019.

[23] M. Kaur, L. Kansal, N. Kaur, G. S. Gaba, and D. P. Agrawal, “Analysis of Image Transmission using MIMO-Alamouti Space-time Encoding," Int. J. Advanced Sci. Eng. Information Technol., vol. 9, no. 1, pp. 39-45, 2019.

[24] I. Pasya, T. Kobayashi, M. F. Abdul Khalid, N. A. Wahab, N. E. A. Rashid, and Z. Awang, "Target localization in MIMO OFDM Radars Adopting Adaptive Power Allocation Among Selected Sub-carriers," Int. J. Advanced Sci. Eng. Information Technol., vol. 7, no. 1, pp. 291 298, 2017. 